\title{
Erythrocyte-Rich Thrombus Is Associated with Reduced Number of Maneuvers and Procedure Time in Patients with Acute Ischemic Stroke Undergoing Mechanical Thrombectomy
}

\author{
Kota Maekawa $^{a}$ Masunari Shibata $^{a}$ Hideki Nakajimab $^{\mathrm{b}}$ Akane Mizutanic $^{\mathrm{c}}$ \\ Yotaro Kitano $^{b}$ Masaru Seguchia $^{a}$ Masayoshi Yamasakic \\ Kazuto Kobayashi $^{a}$ Takanori Sano $^{b}$ Genshin Mori $^{b}$ Tadashi Yabanad \\ Yutaka Naito $^{c}$ Shigetoshi Shimizu $^{\text {b }}$ Fumitaka Miya $^{b}$ \\ a Department of Neuroendovascular Therapy, Ise Red Cross Hospital, Ise City, Japan; \\ ${ }^{b}$ Department of Neurosurgery, Ise Red Cross Hospital, Ise City, Japan; ' Department of \\ Neurology, Ise Red Cross Hospital, Ise City, Japan; d Department of Pathology, Ise Red Cross \\ Hospital, Ise City, Japan
}

\section{Keywords}

Acute ischemic stroke - Computed tomography · Endovascular revascularization · Pathology .

Thrombus formation

\section{Abstract}

Background: Only few studies have investigated the relationship between the histopathology of retrieved thrombi and clinical outcomes. This study aimed to evaluate thrombus composition and its association with clinical, laboratory, and neurointerventional findings in patients treated by mechanical thrombectomy due to acute large vessel occlusion. Methods: At our institution, 79 patients were treated by mechanical thrombectomy using a stent retriever and/or aspiration catheter between August 2015 and August 2016. The retrieved thrombi were quantitatively analyzed to quantify red blood cells, white blood cells, and fibrin by area. We divided the patients into two groups - a fibrin-rich group and an erythrocyte-rich group based on the predominant composition in the thrombus. The groups were compared for imaging, clinical, and neurointerventional data. Results: The retrieved thrombi from 43 patients with acute stroke from internal carotid artery, middle cerebral artery, or basilar artery occlusion were histologically analyzed. Erythrocyte-rich thrombi were present in 18 cases, while fibrin-rich thrombi were present in 25 cases. A cardioembolic etiology was significantly more 
prevalent among the patients with fibrin-rich thrombi than among those with erythrocyterich thrombi. Attenuation of thrombus density as shown on computed tomography images was greater in patients with erythrocyte-rich thrombi than in those with fibrin-rich thrombi. All other clinical and laboratory characteristics remained the same. Patients with erythrocyterich thrombi had a smaller number of recanalization maneuvers, shorter procedure times, a shorter time interval between arrival and recanalization, and a higher percentage of stent retrievers in the final recanalization procedure. The occluded vessels did not differ significantly. Conclusions: In this study, erythrocyte-rich thrombus was associated with noncardioembolic etiology, higher thrombus density, and reduced procedure time.

(C) 2018 The Author(s)

Published by S. Karger AG, Basel

\section{Introduction}

Five randomized trials have demonstrated the benefit of thrombectomy with a stent retriever or flexible aspiration catheter over medical therapy alone in patients with emergency large vessel occlusion [1-6]. Mechanical thrombectomy is increasingly being used for treating acute large vessel occlusion as the first line of endovascular therapy. The use of mechanical thrombectomy devices allows for the retrieval of cerebral thrombi from intracranial vessels and subsequent histopathological analysis. Several studies have suggested that histopathological analysis of a retrieved clot is beneficial in predicting the stroke mechanism in acute large vessel occlusion [7-11]. However, very few studies have investigated the relationship between the histopathology of retrieved thrombi and the clinical outcomes $[7,8$, $10,12]$. A recent study showed that patients with erythrocyte-rich thrombi had higher successful recanalization rates using a MERCI retriever and aspiration catheter [12]. At the present time, thrombectomy with a stent retriever is performed as first-line treatment for patients with acute large vessel occlusion, and has a significantly high rate of successful recanalization. Therefore, clarifying the association between the histopathology of retrieved thrombi and the procedure time is important.

The aim of this study was to evaluate thrombus composition and its association with clinical, laboratory, and neurointerventional findings in patients treated by mechanical thrombectomy due to acute large vessel occlusion.

\section{Subjects and Methods}

\section{Patients}

Patients with acute large vessel occlusion treated in our hospital by mechanical thrombectomy using a stent retriever and/or aspiration catheter due to internal carotid artery, middle cerebral artery, or basilar artery occlusion from August 2015 to August 2016 were included in this study. The patients underwent a nonenhanced computed tomography (CT) scan and a perfusion CT scan before endovascular therapy.

Subtypes of ischemic stroke were determined according to the international Trial of Org 10172 in Acute Stroke Treatment (TOAST) classification. Large artery atherosclerosis was defined as angiographic findings of either significant ( $>50 \%$ ) stenosis or occlusion of a major cervical artery or intracranial artery ipsilateral to the symptomatic hemisphere without evidence of potential sources of cardioembolism in other diagnostic studies. Cardioembolism was defined as at least one cardiac source for an embolus identified in the absence of significant ipsilateral stenosis. 


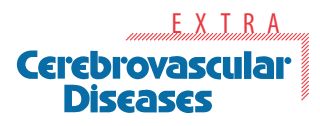

Ceiebrovasc
Diseases
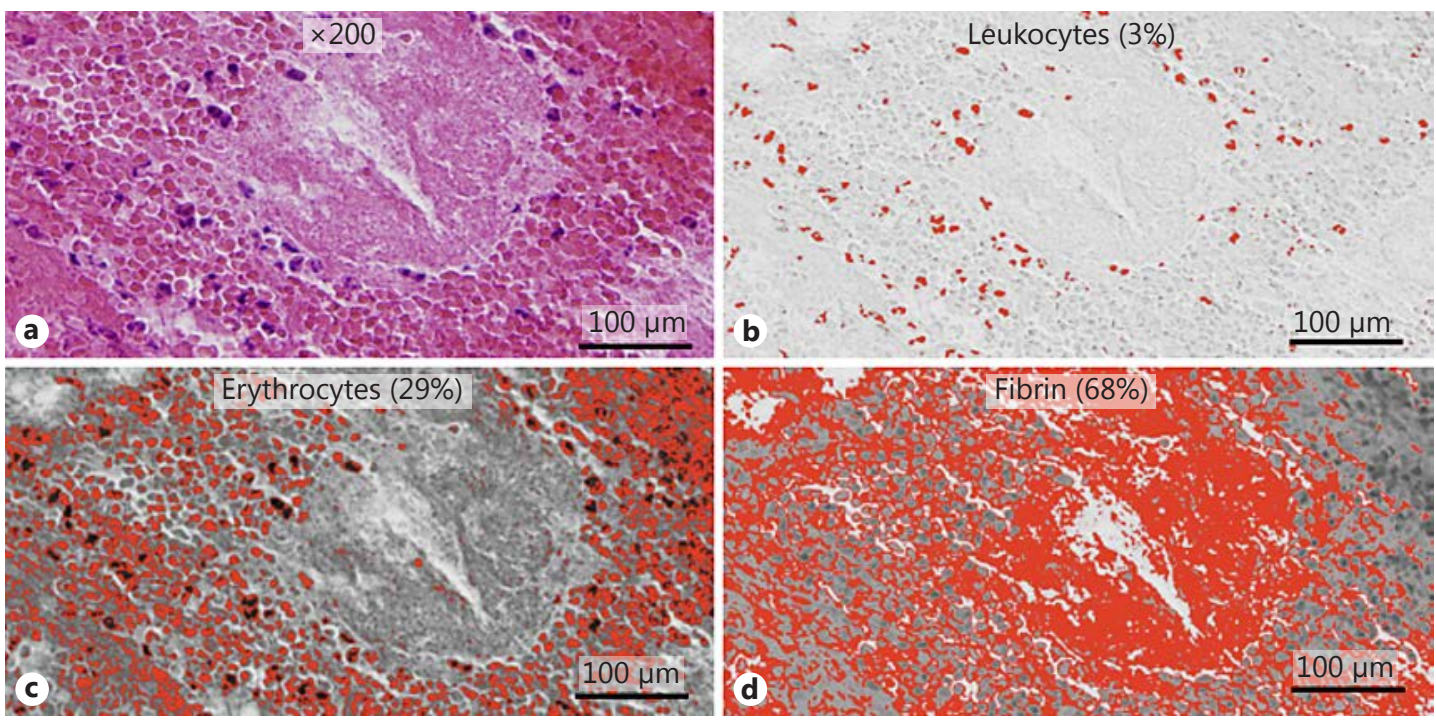

Fig. 1. Histopathological analysis of a retrieved thrombus using the ImageJ software. a Pathology image of a thrombus stained with hematoxylin-eosin. b Leukocytes are depicted in red. Leukocytes represent 3\% of the total composition in this image. c Erythrocytes are depicted in red. Erythrocytes represent $29 \%$ of the total composition in this image. d Fibrin is depicted in red. Fibrin represents $68 \%$ of the total composition in this image. These procedures were performed on all parts of the thrombus. This thrombus was finally categorized as fibrin rich.

The institutional ethics committee approved this study. If a patient had the capacity to consent, written informed consent was obtained from the patient. If this was not the case, written informed consent was obtained from their nearest relatives.

\section{CT Imaging Analysis}

CT imaging examinations were performed using a 64-slice multidetector CT device (GE Healthcare, Little Chalfont, UK). The CT parameters of the noncontrast CT were $120 \mathrm{kVp}, 300$ $\mathrm{mAs}$, and a 5-mm reconstructed slice thickness. For the perfusion CT, $40 \mathrm{~mL}$ of contrast agent (iohexol) was injected into the antecubital vein (20-G needle) at a rate of $4 \mathrm{~mL} / \mathrm{s}$ followed by a 20-mL saline flush at the same rate.

Thrombus attenuation was measured on 3-mm noncontrast CT images in Hounsfield units (HU) by two investigators blinded to any data except the localization of the thrombus. Within the thrombus, a standardized small, round region of interest was drawn, and the mean HU values were used for analysis.

\section{Procedure of Mechanical Thrombectomy}

On admission, a stroke neurologist performed an assessment based on the NIHSS. The inclusion criteria for endovascular thrombectomy were as follows: (1) an NIHSS score $>4$, with no intracranial hemorrhage detected on cranial CT imaging; (2) a small infarct core, defined as an ASPECTS (Alberta Stroke Programme Early CT Score) of 6-10; and (3) a target mismatch pattern on perfusion CT imaging. The occluded vessel was detected on four-dimensional CT angiography.

Cerebral angiography and mechanical recanalization were performed under local anesthesia. In case of agitation, intravenous infusion of propofol was conducted. Stent-based thrombectomy with a Solitaire stent (Covidien, Irvine, CA, USA), a Trevo retriever (Stryker, Kalamazoo, MI, USA), or Revive (Codman Neuro, Raynham, MA, USA) was performed as the 


\section{Cerebrovascular \\ Diseases}

Fig. 2. Histopathology of erythrocyte-rich and fibrin-rich thrombi. $\times 200$. a Erythrocyte-rich thrombus displaying a predominance of erythrocyte infiltration and lower fibrin infiltration. b Fibrin-rich thrombus displaying a predominance of organized fibrin infiltration with few cellular elements.

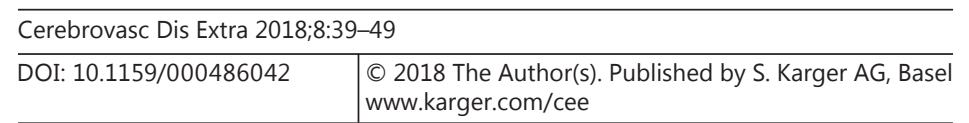

Maekawa et al.: Erythrocyte-Rich Thrombus Is Associated with Reduced Number of Maneuvers and Procedure Time in Patients with Acute Ischemic Stroke

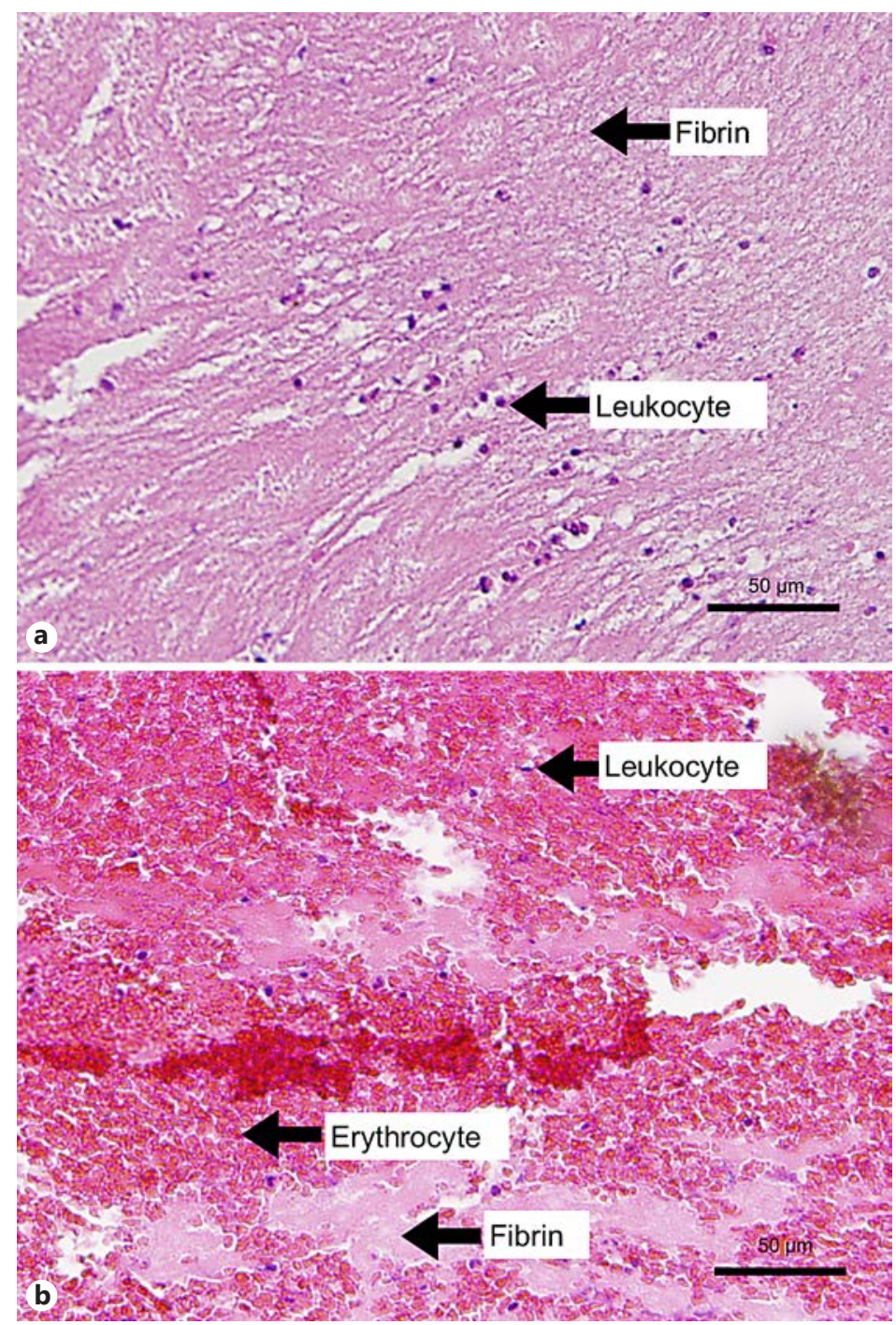

first-line endovascular treatment. When the location of a thrombus was M1 distal or M2, a Penumbra 5MAX ACE (Penumbra, Alameda, CA, USA) was used as an intermediate catheter. When the stent-based thrombectomy was unsuccessful, additional approaches were performed, including using a Penumbra reperfusion catheter and intra-arterial injection of urokinase. The recanalization status was assessed on the final angiogram and was classified according to the modified TICI (Thrombolysis in Cerebral Infarction) scale, with successful recanalization defined as a modified TICI grade of $2 \mathrm{~b}$ or 3 . Clinical outcome was assessed by a stroke neurologist using the modified Rankin Scale (mRS) at 90 days after treatment. A good outcome was defined as an mRS score of $0-2$ or no change. A patient was excluded due to death from lung cancer 2 months after thrombectomy.

\section{Histopathological Analysis}

The retrieved thrombus material was fixed in 10\% neutralized buffered formalin immediately after retrieval. The formalin-fixed, paraffin-embedded thrombus was cut at 2- $\mu \mathrm{m}$ thickness. All thrombi were stained with hematoxylin-eosin. The slides with the stained specimens were scanned at $\times 200$ with a DP 72 device (Olympus, Tokyo, Japan) and digitally stored. 

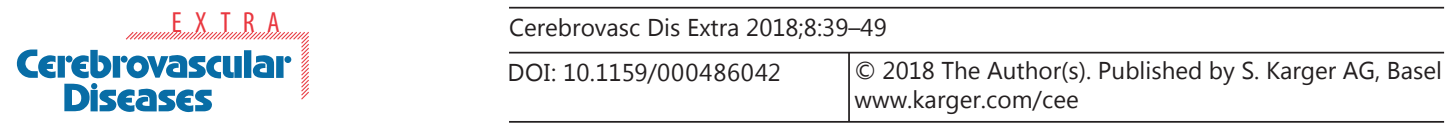

Maekawa et al.: Erythrocyte-Rich Thrombus Is Associated with Reduced Number of Maneuvers and Procedure Time in Patients with Acute Ischemic Stroke

Table 1. Differences in preprocedural and procedural parameters between the two groups

\begin{tabular}{|c|c|c|c|}
\hline & $\begin{array}{l}\text { Erythrocyte rich } \\
(n=18)\end{array}$ & $\begin{array}{l}\text { Fibrin rich } \\
(n=25)\end{array}$ & $\begin{array}{l}p \\
\text { value }\end{array}$ \\
\hline Age, years & $75.2 \pm 14$ & $77.6 \pm 13.9$ & 0.58 \\
\hline Female, $n(\%)$ & $9(50)$ & $13(52)$ & 1 \\
\hline Baseline NIHSS score & $20.3 \pm 9.2$ & $20.2 \pm 6.3$ & 0.97 \\
\hline Diabetes mellitus, $n(\%)$ & $2(11)$ & $2(8)$ & 1 \\
\hline Hypertension, $n(\%)$ & $6(33)$ & $15(60)$ & 0.16 \\
\hline Anticoagulation agent, $n(\%)$ & $5(28)$ & $6(24)$ & 0.77 \\
\hline Antiplatelet agent, $n(\%)$ & $4(22)$ & $2(8)$ & 0.18 \\
\hline \multicolumn{4}{|l|}{ Stroke etiology according to TOAST criteria, $n(\%)$} \\
\hline Large artery atherosclerosis (TOAST 1) & $3(17)$ & $2(8)$ & 0.63 \\
\hline Cardioembolism (TOAST 2) & $9(50)$ & $21(84)$ & 0.01 \\
\hline Other determined cause (TOAST 4) & $1(6)$ & $0(0)$ & 0.42 \\
\hline Other undetermined cause (TOAST 5) & $5(29)$ & $2(7)$ & 0.07 \\
\hline \multicolumn{4}{|l|}{ Occlusion site, $n(\%)$} \\
\hline ICA & $8(44)$ & $10(40)$ & 1 \\
\hline M1 proximal & $3(17)$ & $5(20)$ & 1 \\
\hline M1 distal & $2(11)$ & $5(19)$ & 0.68 \\
\hline M2 & $2(11)$ & $4(15)$ & 1 \\
\hline Basilar artery & $3(17)$ & $1(4)$ & 0.29 \\
\hline ASPECTS & $9.1 \pm 2.5$ & $8.9 \pm 2.1$ & 0.6 \\
\hline Thrombus density, HU & $48.1(24.7-55.1)$ & $32.1(27-41.8)$ & 0.04 \\
\hline Infusion of tissue plasminogen activator, $n(\%)$ & $10(56)$ & $10(40)$ & 0.49 \\
\hline Symptom onset to puncture time interval, min & $225(85-2,064)$ & $191(60-1,324)$ & 0.65 \\
\hline \multicolumn{4}{|l|}{ Mechanical thrombectomy device, $n(\%)$} \\
\hline Stent retriever & $15(83)$ & $23(92)$ & 0.81 \\
\hline Aspiration catheter & $13(72)$ & $21(84)$ & 0.77 \\
\hline Procedure time, min & $24.5(8-85)$ & $44(13-129)$ & $<0.01$ \\
\hline Arrival-to-recanalization time interval, min & $86.5(32-146)$ & $110(58-222)$ & 0.04 \\
\hline Symptom onset-to-recanalization time interval, min & $256(128-2,100)$ & $268(100-1,367)$ & 0.4 \\
\hline Number of recanalization maneuvers & $1.8 \pm 1.1$ & $2.9 \pm 1.5$ & 0.02 \\
\hline
\end{tabular}

Values are presented as means \pm SD or medians (range) unless specified otherwise. Procedure time is the time interval between catheterization of the balloon guiding catheter and recanalization. NIHSS, National Institutes of Health Stroke Scale; TOAST, Trial of Org 10172 in Acute Stroke Treatment; ICA, internal carotid artery; ASPECTS, Alberta Stroke Programme Early CT Score; HU, Hounsfield units.

The ImageJ software (National Institutes of Health, Bethesda, MD, USA) and Photoshop Elements 14 (Adobe Systems Inc., San Jose, CA, USA) were used for the relative quantitative analysis of erythrocytes, fibrin, and leukocytes using color-based segmentation (Fig. 1). Thrombi were classified as erythrocyte rich or fibrin rich according to their predominant composition (Fig. 2). The histopathological analysis was performed by an experienced pathologist and an investigator blinded to the clinical and interventional data. The histopathological assessment included organization, degree of the main composition, presence of calcifications, cholesterol crystals, and endothelial cells.

\section{Statistical Analysis}

The baseline characteristics are summarized as means (with standard deviation [SD]), medians (with range), or frequency counts and proportions. For comparisons between categorical variables, the $\chi^{2}$ test was used. The Student $t$ test was used in case of variables with a normal distribution. The Mann-Whitney U test was used in case of variables with a nonnormal distribution. The two-sided Spearman coefficient was used to express correlations between 

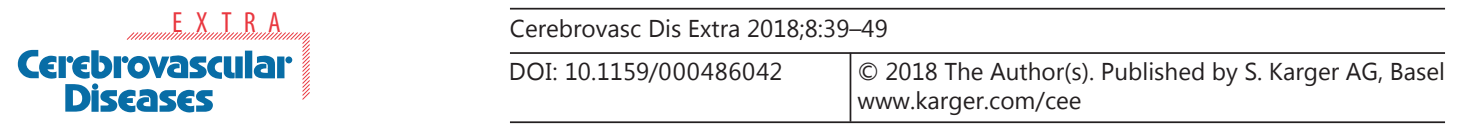

Maekawa et al.: Erythrocyte-Rich Thrombus Is Associated with Reduced Number of Maneuvers and Procedure Time in Patients with Acute Ischemic Stroke

Table 2. Differences in final recanalization procedures between the study groups

\begin{tabular}{llll}
\hline & $\begin{array}{l}\text { Erythrocyte rich } \\
(n=18)\end{array}$ & $\begin{array}{l}\text { Fibrin rich } \\
(n=25)\end{array}$ & $p$ value \\
\hline Stent retriever & $13(72 \%)$ & $9(36 \%)$ & 0.04 \\
Aspiration catheter & $4(22 \%)$ & $14(56 \%)$ & 0.06 \\
$\quad$ Adapt first & $1(6 \%)$ & $2(8 \%)$ & 1 \\
$\quad$ Stent first, adapt second & $1(6 \%)$ & $8(32 \%)$ & 0.08 \\
$\quad$ For distal migration & $2(11 \%)$ & $4(16 \%)$ & 1 \\
Intra-arterial injection of urokinase & $1(6 \%)$ & $2(8 \%)$ & 1 \\
\hline
\end{tabular}

specific thrombus parameters and clinical and interventional parameters. The statistical analysis was performed using EZR (Saitama Medical Center, Jichi Medical University, Saitama, Japan) and SPSS (version 24.0; SPSS, Inc., Chicago, IL, USA). $p$ values $<0.05$ were considered significant. All analyses were retrospective and performed in an explorative manner.

\section{Results}

During the study period, 79 consecutive patients were treated in our hospital by mechanical thrombectomy using a stent retriever and/or aspiration catheter due to internal carotid artery, middle cerebral artery, or basilar artery occlusion. In 6 patients, no recanalization could be achieved (TICI score 0 ), whereas 2 patients reached a TICI score of $2 \mathrm{a}$, and 71 patients reached a TICI score of $2 \mathrm{~b}$ or 3 . In 36 patients, no evaluable thrombus material could be retrieved. Thrombi were available for analysis in 43 cases ( 22 female, aged 43-95 years, median 76), with erythrocyte-rich thrombi present in $42 \%$ (18/43) of the cases and fibrinrich thrombi present in 58\% (25/43) of the cases.

\section{Preprocedural and Procedural Parameters}

The clinical characteristics were generally similar (Table 1), but the patients with fibrinrich thrombi had a significantly higher percentage of cardioembolism and lower thrombus density in HU when compared with the patients with erythrocyte-rich thrombi (Table 1). There was a weak positive correlation between erythrocyte and thrombus attenuation $(R=$ $0.30, p=0.06$ ). There was no significant difference in the percentage of recombinant tissue plasminogen activator infusion (Table 1).

The patients with erythrocyte-rich thrombi required a smaller number of recanalization maneuvers ( $1.8 \pm 1.1$ vs. $2.9 \pm 1.5 ; p=0.02$ ), had a shorter procedure time (median $24.5 \mathrm{~min}$ [8-85] vs. $44 \min$ [13-129]; $p<0.01$ ), and had a shorter arrival-to-recanalization time interval (median $86.5 \min$ [32-146] vs. $110 \mathrm{~min}$ [58-222]; $p=0.04$ ). The patients with erythrocyterich thrombi had a higher percentage of stent retrievers in the final recanalization procedure (72 vs. $36 \% ; p=0.04$ ) (Table 2 ).

Procedure time was correlated with erythrocyte infiltration $(R=-0.34 ; p=0.02)$ and fibrin infiltration $(R=0.35 ; p=0.02$ ) (Fig. 3). There was no significant correlation between procedure time and leukocyte infiltration $(R=0.25$; $p=0.11$ ) (Fig. 3 ).

\section{Outcome Measures}

A patient with a fibrin-rich thrombus had a TICI score of $2 \mathrm{a}$, the other patients had TICI scores of $2 \mathrm{~b}$ or 3 . The patients with erythrocyte-rich thrombi had a tendency for a more favorable clinical outcome (mRS score $0-2$ or no change at 90 days; 71 vs. $36 \% ; p=0.06$ ) (Table 3 ). 
Table 3. Differences in outcome measures between the two groups

\begin{tabular}{llll}
\hline & $\begin{array}{l}\text { Erythrocyte rich } \\
(n=18)\end{array}$ & $\begin{array}{l}\text { Fibrin rich } \\
(n=25)\end{array}$ & $p$ value \\
\hline TICI class 2b or 3 & $18(100 \%)$ & $\begin{array}{c}24(96 \%) \\
9(36 \%)\end{array}$ & $\begin{array}{l}1 \\
\text { mRS score } 0-2 \text { or no change at } 90 \text { days }\end{array}$ \\
\hline
\end{tabular}

TICI, Thrombolysis in Cerebral Infarction; mRS, modified Rankin Scale. ${ }^{1} n=17$.

Fig. 3. Procedure time was correlated with erythrocyte infiltration (a), fibrin infiltration (b), and leukocyte infiltration (c).
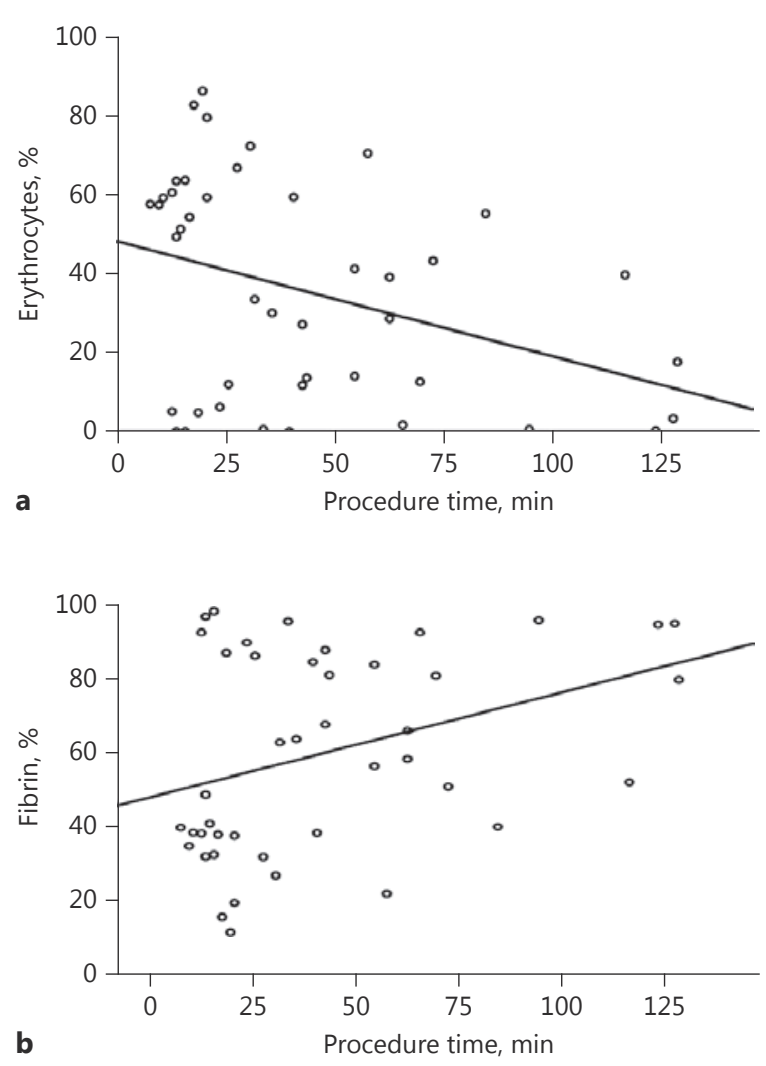

b

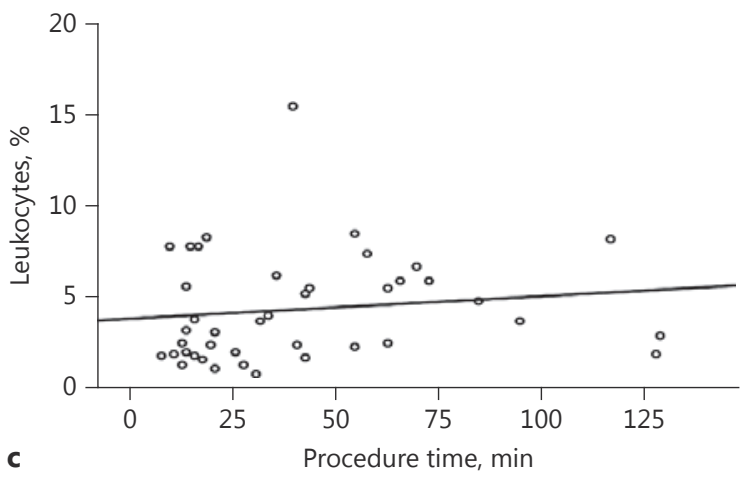


Maekawa et al.: Erythrocyte-Rich Thrombus Is Associated with Reduced Number of

Fig. 4. Differences in mean percentages of thrombus composition by Trial of Org 10172 in Acute Stroke Treatment (TOAST) class. Leukocyte (a), erythrocyte (b), and fibrin infiltration $(\mathbf{c})$.
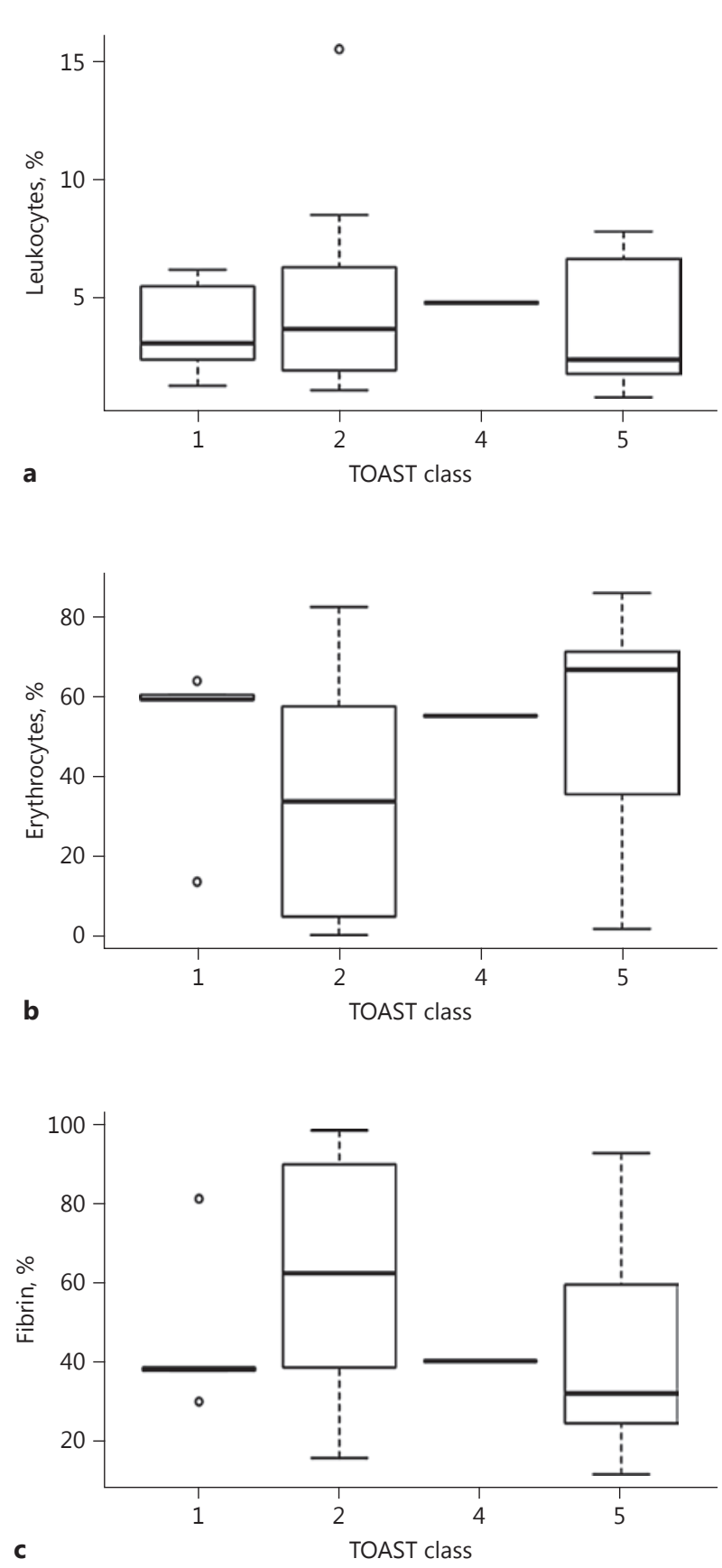

Histopathological Findings

All clots displayed a heterogeneous pattern of three different components: fibrin, erythrocytes, and leukocytes. There were no cholesterol crystals, calcified materials, smoothmuscle cells, or other components of the intima or vessel wall structures.

The subtypes of stroke were classified as large artery atherosclerosis (TOAST 1) in 5 cases (12\%), cardioembolism (TOAST 2) in 30 cases (70\%), another determined etiology (TOAST 4) in 1 case (2\%), and an undetermined etiology (TOAST 5) in 7 cases (16\%). A single 
patient in TOAST class 4 had a vertebral artery dissection. Of the 7 patients classified in TOAST class 5, 1 patient had atrial fibrillation and lung cancer, and 6 patients were diagnosed with cryptogenic stroke. Transesophageal echocardiography was performed in all of the cases classified as cryptogenic.

\section{Stroke Etiology}

The clots in the patients with a cardioembolism contained a higher proportion of fibrin ( $66.2 \pm 25.8$ vs. $46.4 \pm 25.5 \% ; p=0.03)$ and a lower proportion of erythrocytes $(29.5 \pm 26.2$ vs. $49.6 \pm 26.1 \% ; p=0.03$ ) than those in the patients with no cardioembolism (Fig. 4). The large artery atherosclerotic and cryptogenic thrombi had a higher percentage of erythrocytes. There was no significant difference in the percentage of leukocytes between the patients with cardioembolism and those with no cardioembolism.

\section{Discussion}

This study shows that (1) erythrocyte-rich thrombi were associated with a smaller number of recanalization maneuvers, shorter procedure time, and shorter arrival-to-recanalization time interval, (2) erythrocyte-rich thrombi have a higher thrombus density in $\mathrm{HU}$, and (3) cardioembolic thrombi have a higher percentage of fibrin and fewer erythrocytes than noncardioembolic thrombi.

Interestingly, a significant relationship between thrombus composition and clinical outcome was observed. Erythrocyte-rich thrombi were associated with a smaller number of recanalization maneuvers, shorter procedure time, and shorter arrival-to-recanalization time interval. At our institution, we perform mechanical thrombectomy with a stent retriever as the first-line therapy. Patients with erythrocyte-rich thrombi had successful recanalization using the stent retriever, whereas those with fibrin-rich thrombi had failed recanalization using a stent retriever and underwent successful recanalization using an aspiration catheter. To date, very few studies have investigated the relationship between the histopathology of retrieved thrombi and clinical outcomes. One recent study described that patients with erythrocyte-rich thrombi had a higher successful recanalization rate with a MERCI retriever and an aspiration catheter [12]. Moreover, in a stent retriever-based study, thrombus density measured in HU (undoubtedly reflecting higher erythrocyte infiltration) was strongly predictive of successful recanalization [13]. Recently, thrombectomy with a stent retriever has been performed as first-line therapy for patients with acute large vessel occlusion, and it had a significantly higher rate of successful recanalization.

Patients with erythrocyte-rich thrombi had a shorter arrival-to-recanalization time interval and had more favorable clinical outcomes, whereas there was no significant difference in symptom onset-to-recanalization time interval. Recent publications have described a stronger association of good outcome with the time interval from arrival at the emergency department to recanalization, but not with the time interval from symptom onset to arrival at the emergency department [14]. This may be because the inclusion criteria eliminated patients with clinical features of a very mild ischemic injury, clinical and brain imaging features that indicated an advanced and extensive injury, or an unknown or undocumented time of stroke onset. In contrast, the time of arrival at the emergency department is generally accurately documented in patients' medical records [15]. In some patients, symptom onset occurs during sleep and the actual time of symptom onset is not known.

Several studies have investigated a possible relationship between the histology of retrieved thrombi and stroke etiology [7-11,16,17]. In our study, we found that the proportion of fibrin in retrieved thrombi was higher in patients with cardioembolism than in those with 
no cardioembolism. This is in line with the findings of Boeckh-Behrens et al. [7, 8] and Niesten et al. [10]. They reported that cardioembolic thrombi were more organized than noncardioembolic thrombi. Fresh erythrocyte-rich thrombi may occur around the local plaque rupture, or organized thrombi can embolize to the brain incompletely, which may ultimately lead to complete thrombosis [7]. Our results seem to be in contrast to a published study by Ha et al. [9], questioning the traditional concept of cardioembolic thrombi being mostly erythrocyterich thrombi. A recent systematic review showed no association between thrombus composition and etiology [17]. All previous studies that reported a correlation between thrombus histology and stroke etiology used hematoxylin-eosin staining to evaluate thrombus composition. They reported that hematoxylin-eosin staining characteristics did not provide enough information to understand the association between thrombus composition and stroke etiology, and immunostaining was required to further understand it.

Our study shows that erythrocyte-rich thrombi have a higher density on CT than fibrinrich thrombi. Pranevicius et al. [16] reported that the mean percentage of erythrocytes was greater in thrombi with hyperdense middle cerebral artery signs. Niesten et al. [18] showed a relationship between higher thrombus density and noncardioembolism. On the other hand, the susceptibility vessel sign on $\mathrm{T} 2 *$-weighted gradient echo imaging or susceptibilityweighted imaging, which reflects a high amount of erythrocytes, was related to cardioembolism $[9,16,19]$. This discrepancy may be due to a thrombus's age. These sequences detect the paramagnetic effects of deoxyhemoglobin and methemoglobin. Fresh erythrocyte-rich thrombi still contain significant amounts of diamagnetic oxyhemoglobin, while old cardioembolic thrombi have time to become fully deoxygenated and paramagnetic $[20,21]$.

Our study has several limitations. Only a relatively small number of thrombi were investigated, making it difficult to draw any strong conclusions. There may be a selection bias toward more stable thrombi, as not all thrombi or thrombus fragments retrieved could be included in the final analysis due to disintegration during retrieval or histological processing. In addition, we investigated patients with acute large vessel occlusion in 54\% of all cases treated in our hospital. Furthermore, patients with investigated thrombi had successful reperfusion (TICI 2 b or 3 ) in $96 \%$ of the cases. Thus, we could not evaluate retrieved thrombi from patients with unsuccessful reperfusion.

\section{Conclusion}

Erythrocyte-rich thrombi were associated with a reduced number of recanalization maneuvers, shorter arrival-to-recanalization time interval, and more favorable clinical outcomes. This study suggests that the histological composition of retrieved thrombi is significantly different between stroke subtypes. Cardioembolic thrombi have a higher percentage of fibrin and fewer erythrocytes than do noncardioembolic thrombi. In addition, a high thrombus density in HU is strongly associated with erythrocyte-rich thrombi. These findings will have to be validated in future studies.

\section{Acknowledgments}

The authors would like to thank Enago (www.enago.jp) for the English language review. 


\title{
Disclosure Statement
}

\author{
The authors have no conflicts of interest to disclose.
}

\section{References}

1 Berkhemer OA, Fransen PS, Beumer D, van den Berg LA, Lingsma HF, Yoo AJ, et al; MR CLEAN Investigators: A randomized trial of intraarterial treatment for acute ischemic stroke. N Engl J Med 2015;372:11-20.

-2 Campbell BC, Mitchell PJ, Kleinig TJ, Dewey HM, Churilov L, Yassi N, et al; EXTEND-IA Investigators: Endovascular therapy for ischemic stroke with perfusion-imaging selection. N Engl J Med 2015;372:1009-1018.

-3 Goyal M, Demchuk AM, Menon BK, Eesa M, Rempel JL, Thornton J, et al; ESCAPE Trial Investigators: Randomized assessment of rapid endovascular treatment of ischemic stroke. N Engl J Med 2015;372:1019-1030.

4 Jovin TG, Chamorro A, Cobo E, de Miquel MA, Molina CA, Rovira A, et al; REVASCAT Trial Investigators: Thrombectomy within 8 hours after symptom onset in ischemic stroke. N Engl J Med 2015;372:2296-2306.

-5 Saver JL, Goyal M, Bonafe A, Diener HC, Levy EI, Pereira VM, et al; SWIFT PRIME Investigators: Stent-retriever thrombectomy after intravenous t-PA vs t-PA alone in stroke. N Engl J Med 2015;372:2285-2295.

6 Goyal M, Menon BK, van Zwam WH, Dippel DW, Mitchell PJ, Demchuk AM, et al; HERMES Collaborators: Endovascular thrombectomy after large-vessel ischaemic stroke: a meta-analysis of individual patient data from five randomised trials. Lancet 2016;387:1723-1731.

7 Boeckh-Behrens T, Kleine JF, Zimmer C, Neff F, Scheipl F, Pelisek J, Schirmer L, Nguyen K, Karatas D, Poppert H: Thrombus histology suggests cardioembolic cause in cryptogenic stroke. Stroke 2016;47:1864-1871.

-8 Boeckh-Behrens T, Schubert M, Förschler A, Prothmann S, Kreiser K, Zimmer C, Riegger J, Bauer J, Neff F, Kehl V, Pelisek J, Schirmer L, Mehr M, Poppert H: The impact of histological clot composition in embolic stroke. Clin Neuroradiol 2016;26:189-197.

-9 Ha EJ, Baek JH, Na DG, Kim JH, Kim JK, Min HS, Song DE, Lee KE, Shong YK: The role of core needle biopsy and its impact on surgical management in patients with medullary thyroid cancer: clinical experience at 3 medical institutions. AJNR Am J Neuroradiol 2015;36:1512-1517.

10 Niesten JM, van der Schaaf IC, van Dam L, Vink A, Vos JA, Schonewille WJ, de Bruin PC, Mali WP, Velthuis BK: Histopathologic composition of cerebral thrombi of acute stroke patients is correlated with stroke subtype and thrombus attenuation. PLoS One 2014;9:e88882.

11 Simons N, Mitchell P, Dowling R, Gonzales M, Yan B: Thrombus composition in acute ischemic stroke: a histopathological study of thrombus extracted by endovascular retrieval. J Neuroradiol 2015;42:86-92.

12 Hashimoto T, Hayakawa M, Funatsu N, Yamagami H, Satow T, Takahashi JC, Nagatsuka K, Ishibashi-Ueda H, Kira JI, Toyoda K: Histopathologic analysis of retrieved thrombi associated with successful reperfusion after acute stroke thrombectomy. Stroke 2016;47:3035-3037.

13 Mokin M, Morr S, Natarajan SK, Lin N, Snyder KV, Hopkins LN, Siddiqui AH, Levy EI: Thrombus density predicts successful recanalization with Solitaire stent retriever thrombectomy in acute ischemic stroke. J Neurointerv Surg 2015; 7:104-107.

14 Saver JL, Goyal M, van der Lugt A, Menon BK, Majoie CB, Dippel DW, et al; HERMES Collaborators: Time to treatment with endovascular thrombectomy and outcomes from ischemic stroke: a meta-analysis. JAMA 2016;316:1279-1288.

-15 Spokoyny I, Raman R, Ernstrom K, Kim AJ, Meyer BC, Karanjia NP: Accuracy of first recorded "last known normal" times of stroke code patients. J Stroke Cerebrovasc Dis 2015;24:2467-2473.

-16 Pranevicius O, Pranevicius M, Liebeskind DS: Partial aortic occlusion and cerebral venous steal: venous effects of arterial manipulation in acute stroke. Stroke 2011;42:1478-1481.

17 Brinjikji W, Duffy S, Burrows A, Hacke W, Liebeskind D, Majoie C, Dippel DWJ, Siddiqui AH, Khatri P, Baxter B, Nogeuira R, Gounis M, Jovin T, Kallmes DF: Correlation of imaging and histopathology of thrombi in acute ischemic stroke with etiology and outcome: a systematic review. J Neurointerv Surg 2017;9:529-534.

-18 Niesten JM, van der Schaaf IC, Biessels GJ, van Otterloo AE, van Seeters T, Horsch AD, et al; Dutch Acute Stroke Trial (DUST): Relationship between thrombus attenuation and different stroke subtypes. Neuroradiology 2013;55:1071-1079.

19 Horie N, Tateishi Y, Morikawa M, Morofuji Y, Hayashi K, Izumo T, Tsujino A, Nagata I, Matsuo T: Acute stroke with major intracranial vessel occlusion: characteristics of cardioembolism and atherosclerosis-related in situ stenosis/occlusion. J Clin Neurosci 2016;32:24-29.

20 Kidwell CS, Chalela JA, Saver JL, Starkman S, Hill MD, Demchuk AM, Butman JA, Patronas N, Alger JR, Latour LL, Luby ML, Baird AE, Leary MC, Tremwel M, Ovbiagele B, Fredieu A, Suzuki S, Villablanca JP, Davis S, Dunn B, Todd JW, Ezzeddine MA, Haymore J, Lynch JK, Davis L, Warach S: Comparison of MRI and CT for detection of acute intracerebral hemorrhage. JAMA 2004;292:1823-1830.

-21 Linfante I, Llinas RH, Caplan LR, Warach S: MRI features of intracerebral hemorrhage within $2 \mathrm{~h}$ from symptom onset. Stroke 1999;30:2263-2267. 